\section{Medidas de salud mental y bienestar subjetivo en una muestra de hombres gays $y$ mujeres lesbianas en Chile}

\author{
JAIME BARRIENTOS ${ }^{1, \mathrm{a}}$, FABIOLA GÓMEZ ${ }^{2, \mathrm{~b}}$, \\ MANUEL CÁRDENAS ${ }^{3, \mathrm{c}}$, MÓNICA GÚZMAN $^{4, \mathrm{~d}}$, \\ JOAQUÍN BAHAMONDES ${ }^{5, \mathrm{e}}$
}

\section{Health and wellbeing of sexual minorities}

Background: Most of the information in Chile about health and wellbeing of sexual minorities refers to risk behaviors. Aim: To assess health and wellbeing in a sample of Chilean homosexual men and women. Material and Methods: Spanish versions of the Satisfaction With Life Scale and Outcome Questionnaire-45 (OQ45) were answered by 191 homosexual women and 256 homosexual men aged 18 to 67 years, from four Chilean cities. Results: Lesbian women have better levels of satisfaction with life and adjustment in personal relationships than homosexual men. Eight percent of respondents had suicidal thoughts in some moment of their life. Conclusions: The information gathered in this work could help in the development of mental health policies for sexual minorities.

(Rev Med Chile 2017; 145: 1115-1121)

Key words: Homosexuality; Mental Health; Sexual Minorities; Suicida Ideation.
'Escuela de Psicología, Universidad de Santiago de Chile. Santiago, Chile.

2Escuela de Psicología, Facultad de Ciencias Sociales, Pontificia Universidad Católica de Chile. Santiago, Chile.

${ }^{3}$ Escuela de Psicología, Facultad de Medicina, Universidad de Valparaíso. Valparaíso, Chile. ${ }^{4}$ Escuela de Psicología, Facultad de Humanidades, Universidad Católica del Norte. Antofagasta, Chile.

${ }^{5}$ Escuela de Psicología, Facultad de Ciencias,

University of Auckland. Auckland, Nueva Zelanda.

a Psicólogo, PhD en Psicología Social.

bPsicóloga, Magister en Psicología Social, estudiante de Doctorado en Psicología, Escuela de Psicología, Pontificia Universidad Católica de Chile, Santiago, Chile.

'Psicólogo, PhD en Psicología Social.

dPsicóloga, PhD en Psicología.

ePsicólogo, Magíster en Psicología Social, estudiante de Doctorado en Psicología, Escuela de Psicología, University of Auckland, Auckland, Nueva Zelanda.

Apoyo financiero: Este artículo contó con el apoyo del Proyecto Fondecyt 1110423, titulado "Homofobia y efectos psicosociales en la calidad de vida de homosexuales en Chile: Hacia un modelo comprensivo." La organización que proporcionó el apoyo no tuvo influencia en el diseño del estudio; en la recolección, análisis o interpretación de los datos; en la preparación, revisión o aprobación del manuscrito.

Recibido el 6 de diciembre de 2016, aceptado el 7 de septiembre de 2017.

Correspondencia a:

Jaime Barrientos

Escuela de Psicología, Universidad de Santiago de Chile. Santiago, Chile.

jaime.barrientos@usach.cl
$\mathrm{E}$ n las últimas décadas se han observado cambios positivos en el reconocimiento de los derechos de las minorías sexuales en Chile ${ }^{1}$. Sin embargo, Cárdenas y Barrientos han propuesto que, en la actualidad, la expresión de la homofobia a tomado una forma más sutil y menos explícita que antaño ${ }^{2}$. Por su parte, Barrientos y Bozon, reportan que cerca de $80 \%$ de las personas lesbianas, gay, bisexuales y transgénero (LGBT) encuestadas, en el contexto de la Marcha del Orgullo LGBT chileno realizada en el 2011, reportan haber sido víctimas de discriminación y agresión ${ }^{3}$. Según el modelo de estrés de las minorías (en inglés, Minority Stress $)^{4}$, las experiencias de homofobia que las minorías sexuales deben enfrentar en su vida cotidiana pueden tener un efecto importante en su bienestar y salud mental ${ }^{5}$.

\section{Salud mental y bienestar de hombres gays y mujeres lesbianas}

Diversos estudios han constatado la existencia de síntomas psicológicos en la población homosexual, tales como angustia y estrés ${ }^{6-8}$, mayor prevalencia de conductas de riesgo y vulnerabilidad para la salud física y mental, como comportamientos de riesgo asociados al VIH ${ }^{8-12}$; e intención suicida o suicidio ${ }^{13,14}$. No obstante, la mayor parte de la evidencia empírica corresponde a otros contextos como EE.UU. o Europa ${ }^{8,15-17}$. En América Latina y, 
paritcularmente en Chile son escasos los estudios sobre estos temas y su prevalencia ${ }^{1}$.

Una reciente revisión, que comparó la prevalencia de trastornos mentales entre personas heterosexuales y minorías sexuales, encontró que entre gays, lesbianas y personas bisexuales el riesgo de sufrir depresión o trastornos de ansiedad era 1,5 veces más alto que en personas heterosexuales ${ }^{18}$. Por su parte, otro estudio encontró que hombres gays y bisexuales tenían niveles más altos de depresión, ataques de pánico y malestares psicológicos en comparación a hombres heterosexuales; y que las mujeres lesbianas y bisexuales, en comparación a su contraparte heterosexual, mostraban una mayor prevalencia de ansiedad generalizada ${ }^{19}$.

Con el propósito de avanzar en la generación de conocimiento contextualizado respecto de las minorías sexuales y su bienestar y salud mental, el principal objetivo de este estudio es describir los niveles de bienestar y la salud mental en una muestra de hombres gays y mujeres lesbianas en Chile. Este objetivo es relevante dado que aún persiste violencia hacia la población gay y lésbica en el país, generando efectos negativos en su salud ${ }^{1}$ y que, usualmente el énfasis ha sido puesto únicamente en las consecuencias negativas de dicha violencia.

Dado el carácter exploratorio descriptivo de este estudio, y frente a la escasa evidencia empírica frente al tema, este trabajo no cuenta con hipótesis previas tentativas.

\section{Material y Método}

\section{Participantes y selección de la muestra}

La muestra fue de 467 participantes que se auto-identificaron como hombres gays y mujeres lesbianas, entre 18 y 67 años $(M=27,9$ y $\mathrm{DE}=7,9)$. La muestra quedó constituida por $57,4 \%(n=268)$ de hombres gays, con edades entre 18 y 67 años $(M=27,6 ; D E=7,6)$ y $42,6 \%(n=199)$ de mujeres lesbianas, con edades que van entre 18 y 60 años $(\mathrm{M}=28,1 ; \mathrm{DE}=8,2)$.

Las minorías sexuales son consideradas muestras de difícil acceso ${ }^{20}$, por tanto, no se cuenta con marcos muestrales que permitan seleccionar muestras aleatorias. Por ello, la muestra fue seleccionada a partir de un muestreo en cadena tipo bola de nieve, tal como se ha realizado en estudios previos en la región ${ }^{21,22}$.

Los criterios de selección de la muestra fueron auto-identificación sexual (hombres gays y mujeres lesbianas), tener más de 18 años y ciudad de residencia . Este estudio no consideraba entre sus objetivos el estudio de otras poblaciones.

\section{Medidas}

En primer lugar, incorporamos preguntas de caracterización sociodemográfica. Se preguntó por la auto-identificación sexual como gays o lesbianas, edad (años cumplidos al momento de la entrevista) y ciudad de residencia. Además se indagó sobre la revelación de la orientación sexual de los encuestados (sí/no); a quiénes le ha contado sobre su orientación sexual (opciones de respuesta categórica, Tabla 1); así como si las personas encuestadas tienen pareja (sí/no) y si actualmente viven en pareja (sí/no).

\section{Bienestar subjetivo}

Para medir bienestar subjetivo se utilizó la Escala de Satisfacción con la Vida ${ }^{23}$ en su versión adaptada y validada en Chile ${ }^{24}$. Esta escala consiste en 5 ítems tipo Likert con alternativas de respuesta que van de 1 (completamente en desacuerdo) a 6 (completamente de acuerdo). A mayor puntaje mayor nivel de satisfacción con la vida. Para esta aplicación se obtuvieron buenos niveles de confiabilidad $(\alpha=0,81)$.

\section{Sintomatología socioemocional}

Para evaluar la presencia de sintomatología se utilizó el instrumento Outcome Questionnaire-45 (OQ-45) ${ }^{27}$ en su versión adaptada y estandarizada en población nacional ${ }^{25}$. Esta escala fue diseñada originalmente para evaluar los resultados de la psicoterapia, sin embargo, también permite obtener información acerca de la sintomatología y/o ajuste socioemocional, siendo capaz de discriminar en forma significativa entre muestras clínicas y normativas ${ }^{26}$. La escala evalúa síntomas en los últimos siete días y cuenta con 45 ítems distribuidos en tres dimensiones: Sintomatología ansioso-depresiva, que incluye preguntas acerca de síntomas ansiosos y depresivos, así como algunos reactivos destinados a identificar específicamente conductas de riesgo tales como ideación suicida o abuso de alcohol; Rol Social, que evalúa la presencia de problemas en el ámbito laboral, académico o de ocio; y Relaciones Interpersonales, destinada a identificar dificultades en las relaciones interpersonales significativas para la persona ${ }^{26}$. Las alternativas de 
Tabla 1. Revelación de la orientación sexual y situación afectiva

\begin{tabular}{|c|c|c|c|c|c|c|}
\hline & \multicolumn{2}{|c|}{ Gays } & \multicolumn{2}{|c|}{ Lesbianas } & \multicolumn{2}{|c|}{ Total } \\
\hline & $\mathbf{n}$ & $\%$ & $\mathbf{n}$ & $\%$ & $\mathbf{n}$ & $\%$ \\
\hline \multicolumn{7}{|c|}{ Revelación de la orientación sexual } \\
\hline Sí & 252 & 98,4 & 191 & 95,5 & 443 & 98,9 \\
\hline No & 4 & 1,6 & 1 & 0,5 & 5 & 1,1 \\
\hline Missing data & 12 & & 7 & & 19 & \\
\hline Subtotal & 268 & 100 & 199 & 100 & 467 & 100 \\
\hline \multicolumn{7}{|c|}{ A quiénes ha revelado su orientación sexuala } \\
\hline Padre & 162 & 61,1 & 129 & 65,5 & 291 & 63,0 \\
\hline Madre & 216 & 81,5 & 162 & 82,2 & 378 & 81,8 \\
\hline Hermanos/as & 205 & 77,4 & 152 & 77,2 & 357 & 77,3 \\
\hline Hijos/as & 11 & 4,2 & 18 & 9,1 & 29 & 6,3 \\
\hline Otro/a familiar & 202 & 76,2 & 148 & 75,1 & 350 & 75,8 \\
\hline Compañero/as trabajo/clase & 236 & 89,1 & 154 & 78,2 & 390 & 84,4 \\
\hline Amigos/as gay/lesbianas & 256 & 96,6 & 191 & 97,0 & 447 & 96,8 \\
\hline Amigos/as heterosexuales & 254 & 95,8 & 186 & 94,4 & 440 & 95,2 \\
\hline Otra & 147 & 55,5 & 88 & 44,7 & 235 & 50,9 \\
\hline \multicolumn{7}{|l|}{ Vivir en pareja } \\
\hline Sí & 124 & 46,8 & 135 & 68,2 & 259 & 55,9 \\
\hline No & 141 & 53,2 & 63 & 31,8 & 204 & 44,1 \\
\hline Missing data & 3 & & 1 & & 4 & \\
\hline Subtotal & 268 & 100 & 199 & 100 & 467 & 100 \\
\hline
\end{tabular}

aVariable con respuesta de opción múltiple.

respuesta son tipo Likert con 5 opciones y van de $0=$ Nunca a $4=$ Siempre, donde un puntaje alto total indica mayores niveles de sintomatología socioemocional. El punto de corte para la población chilena es de 73 puntos. Valores superiores a este, dan cuenta de sintomatología socioemocional clínicamente significativa. Para esta aplicación se obtuvo un buen nivel de confiabilidad para la escala global $(\alpha=0,91)$.

\section{Procedimiento}

Este estudio es parte de un proyecto Fondecyt más amplio, reportado en el apoyo financiero, que busca medir la homofobia hacia hombres gay y mujeres lesbianas en Chile y los efectos de estos en la calidad de vida y el bienestar.

El instrumento fue administrado cara a cara, con una duración de 45 min aproximadamente. Antes de ser encuestados, los participantes fueron informados respecto de los objetivos del estudio y firmaron una carta de consentimiento informado, donde aceptaban participar en el estudio y se les aseguraba confidencialidad y anonimato. Este estudio fue aceptado por la Comisión de Ética de la Universidad Católica del Norte.
En base a los criterios de selección, antes señalados, se inició el muestreo con 3 semillas de hombres gays y 3 semillas de mujeres lesbianas, excepto en Santiago donde se comenzó con 6 semillas de hombres gays y 6 semillas de mujeres lesbianas. Cada semilla se seleccionó en base a información entregada por informantes clave. No incluimos a hombres y mujeres bisexuales dada la baja prevalencia descrita previamente en Chile $^{27}$ y dado que este estudio forma parte de un estudio mayor cuyos objetivos no consideraron a este grupo. Luego de ser encuestada cada semilla debía invitar a tres personas gay o lesbianas, que cumplieran los criterios de inclusión, a participar del estudio.

\section{Análisis de datos}

Realizamos análisis descriptivos para cada una de las variables incluidas en el estudio. Además, realizamos análisis de diferencia de medias ( $\mathrm{t}$ de Student) para evaluar posibles diferencias en los indicadores salud mental (OQ-45) y satisfacción con la vida entre hombres gays y mujeres lesbianas. Paralelamente, calculamos el tamaño del efecto para estos análisis. 
Todos los análisis fueron realizados con el software estadístico SPSS, versión 20.0, y G ${ }^{\star}$ Power $3.1^{28}$.

\section{Resultados}

En la Tabla 1 se reportan los resultados para las variables de caracterización social incluidas en el estudio. En relación a la revelación de la orientación sexual, del total de personas encuestadas casi la totalidad de ellas la ha revelado. Si se analiza más detenidamente a qué persona se lo ha contado, la mayor proporción de revelación, tanto para el caso de las mujeres lesbianas como para los hombres gays, es con amigos/as (sobre 94\%). Si se observa la proporción de revelación a los padres, las personas encuestadas reportan una mayor proporción cuando se trata de la madre (82,2\% para mujeres lesbianas y $81,5 \%$ para hombres gays). En el contexto laboral o de estudio, 89,1\% de los hombres gay y 78,2\% de las mujeres lesbianas, han revelado su orientación sexual a compañeros de trabajo o estudio.

Respecto a la situación de pareja, 46,8\% de los hombres gays y $68,2 \%$ de las mujeres lesbianas declara vivir en pareja al momento de la entrevista.

\section{Medidas de bienestar y sintomatología socioemocional}

Se encontraron diferencias estadísticamente significativas en los niveles de satisfacción con la vida entre hombres gays y mujeres lesbianas $\left(t_{(464)}=-2.51, p=.012, d=.23\right)$. Las mujeres lesbianas reportan mayor satisfacción con la vida en comparación con los hombres gays (Tabla 2).

En el caso de la sintomatología medida me- diante la escala OQ-45 se han usado los puntajes de corte establecidos para la población chilena, ya que estos permiten diferenciar la población con o sin malestar clínicamente significativo ${ }^{26}$. Para la escala global, 11,6\% de los hombres gays y $10,6 \%$ de las mujeres lesbianas encuestadas reporta sintomatología socioemocional sobre el punto de corte. Si analizamos la situación por dimensión, constatamos que en la dimensión ansioso-depresivo $9 \%$ de los hombres gays está sobre el puntaje de corte para esta dimensión, así como 11,6\% de las mujeres lesbianas. En la dimensión que explora las relaciones interpersonales $25,4 \%$ de los hombres gays está sobre el puntaje de corte y 16,1\% de las mujeres lesbianas. Finalmente, en la dimensión que evalúa las relaciones sociales, $10,4 \%$ de los hombres gays está sobre el punto de corte en esta dimensión y 13,6\% de las mujeres lesbianas.

Además, se analizó específicamente la pregunta de la escala OQ-45 referida a intención de suicidio. Un 7,9\% del total de la población encuestada ha pensado en quitarse la vida en algún grado considerando para este cálculo solo las alternativas de respuesta "casi nunca", "algunas veces", "casi siempre" o "siempre". En el análisis desagregado por grupo $9 \%$ de los hombres gays y $6,5 \%$ de las mujeres lesbianas ha pensado en quitarse la vida en algún grado.

En cuanto a las diferencias en los niveles de salud mental reportados por hombres gays y mujeres lesbianas, se encontraron diferencias estadísticamente significativas únicamente en la dimensión relaciones interpersonales $\left(t_{(465)}=3,05 ; \mathrm{p}=0,002\right.$, $\mathrm{d}=0,28$ ), siendo las mujeres lesbianas las que reportan menores dificultades en sus relaciones personales cercanas.

Tabla 2. Estadísticos descriptivos para satisfacción con la vida e indicadores de salud mental y diferencia de medias entre hombres gays y mujeres lesbianas

\begin{tabular}{|c|c|c|c|c|c|c|}
\hline Dimensiones & $\begin{array}{c}\text { Gays } \\
\text { M (SD) }\end{array}$ & $\begin{array}{c}\text { Lesbianas } \\
\text { M (SD) }\end{array}$ & $\mathbf{t}$ & df & $\mathbf{p}$ & d \\
\hline Satisfacción con la vida & $4,63 \quad(0,92)$ & $(0,83)$ & $-2,51$ & 464 & 0,012 & 0,23 \\
\hline Sintomatología socioemocionala & $45,19(21,64)$ & $45,59(21,98)$ & $-0,20$ & 465 & 0,842 & 0,02 \\
\hline Sintomatología ansioso depresiva & $23,57 \quad(14,25)$ & $25,62(14,69)$ & $-1,51$ & 465 & 0,131 & 0,14 \\
\hline Relaciones interpersonales & $12,54 \quad(5,98)$ & $(5,63)$ & 3,05 & 465 & 0,002 & 0,28 \\
\hline Rol social & $9,07 \quad(4,04)$ & $(4,34)$ & $-0,06$ & 465 & 0,950 & 0,007 \\
\hline
\end{tabular}

aCorresponde al puntaje de la escala total. Nota. M: media; DE: desviación estándar; t: t de Student; gl: grados de libertad; p: nivel de significancia; d: tamaño del efecto (d de Cohen). 


\section{Discusión}

Este estudio evaluó bienestar y sintomatología socioemocional, es decir, tanto indicadores positivos como negativos de salud. Esto es innovador dado que, usualmente, los estudios en este tema evalúan sólo indicadores negativos de salud. Al respecto, el mayor reporte de satisfacción con la vida de las mujeres lesbianas por sobre hombres gays, difiere de lo reportado para población nacional heterosexual por Vera-Villarroel y colaboradores, donde no se encontraron diferencias entre hombres y mujeres ${ }^{29}$. Esta brecha en la satisfacción vital podría relacionarse al mayor castigo social experimentado por los hombres gays, quienes son un blanco más regular de hostilidad por parte de la población heterosexual en Chile (especialmente masculina $)^{30}$. Asimismo, los hombres gay internalizan más la homofobia que las mujeres lesbianas en nuestro país ${ }^{31}$, al punto que ser gay puede no ser consistente con sus propias expectativas de género, causando un bienestar disminuido en contraste con el de mujeres lesbianas. Futuros estudios deberán profundizar en este tema.

Respecto de la sintomatología socioemocional, el porcentaje de personas homosexuales que reportan malestar significativo es similar a lo reportado en otros estudios con población general que han ocupado el mismo instrumento en Chile ${ }^{32}$. La interpretación de este resultado amerita cautela, pues en este estudio no se comparó explícitamente personas heterosexuales y homosexuales lo que si hizo León y colaboradores, quienes usando el SCL-90 encontraron en adolescentes lesbianas, mayores puntajes de sintomatología clínica que aquellas no lesbianas $^{33}$. No obstante, si comparamos las medias obtenidas en este estudio con otros que han usado la misma escala, encontramos que, en nuestro estudio, todas las medias son inferiores o iguales a las reportadas por el estudio previamente mencionado (sintomatología socioemocional $\mathrm{M}=49$; sintomatología ansioso depresiva $M=29$; relaciones interpersonales $M=11 \mathrm{y} \mathrm{rol}$ social $\mathrm{M}=9)^{34}$. Lo anterior indica que esta población estudiada presenta buenos indicadores de salud, lo que también podría estar asociado a la gran proporción de participantes que ha revelado ya su orientación sexual (casi 99\%), lo que ha sido asociado con cambios beneficiosos en la salud mental, reduciendo la ansiedad y la autoestima ${ }^{35,36}$. Sin embargo, se requieren nuevos estudios en este tema que permitan confirmar y profundizar en estos hallazgos.

Respecto al análisis de la pregunta sobre intención suicida este estudio aporta una de las primeras estimaciones sobre este tema del que no existen reportes previos en el país y la región. El suicidio y la intención suicida constituyen problemas relevantes de salud pública que afectan de modo especial a hombres gays y mujeres lesbianas $^{37}$. Según el modelo del estrés de las minorías, los estresores particulares a la población LGBT se asocia a un incremento del riesgo y probabilidad de intención suicida y suicidio, lo que supone un desafío importante para las políticas de salud pública en contextos en los que aún existe homofobia como lo es el caso chileno ${ }^{1}$.

Pero, también, este estudio constituye, aunque no era su objetivo principal, una aproximación a la descripción sociodemográfica de la población de gays y lesbianas en Chile. Desde nuestro conocimiento, en Chile sólo existe un estudio previo que aporta información al respecto ${ }^{22}$. De este modo, este estudio aporta hallazgos complementarios al estudio mencionado y podría contribuir a la orientación de políticas públicas en salud y ser una línea base para futuros estudios de salud pública en minorías sexuales. A diferencia del trabajo anterior $^{22}$, este incluye a mujeres lesbianas, complementando la información de dicho estudio a través de una muestra más heterogénea.

En cuanto a las limitaciones de este estudio el tipo de muestreo utilizado no permite seleccionar aleatoriamente a los participantes, lo que dificultaría la posibilidad de generalizar estos hallazgos al conjunto de la población gay chilena. Esto es, introduce un sesgo derivado de la selección de la muestra que atenta contra la validez externa. El muestreo en poblaciones de difícil acceso es un desafío para la investigación social, por lo que es necesario seguir avanzando en diseños que incorporen otro tipo de métodos de muestreo, tales como el muestreo conducido por el encuestado ${ }^{38}$ o aquellos basados en los lugares y horarios de reunión de los miembros del grupo ${ }^{39}$.

También, futuros estudios deberían profundizar en la recolección de datos sociodemográficos de la población LGBT. Ya que, si bien existen algunos, estos aún son insuficientes para conocer mejor a esta población y sus necesidades de salud específicas. Del mismo modo, es necesario pro- 
fundizar en los efectos de la homofobia en la salud física y mental de las personas $\mathrm{LGBT}^{40}$.

\section{Referencias}

1. Barrientos J. Violencia homofóbica en America Latina y en Chile. Santiago: Chile: El Buen Aire S.A.; 2015. 154 p.

2. Cardenas M, Barrientos JE. The attitudes toward lesbians and gay men scale (ATLG): adaptation and testing the reliability and validity in Chile. J Sex Res 2008; 45 (2): 140-9.

3. Barrientos J, Bozon MV. Victimization against Gay Men and Lesbians in Chile in the context of the XIII Sexual Diversity Pride Parade -Santiago 2011: Two types of discrimination or just one?. Revista Interdisciplinaria 2014; 31 (2): 323-39.

4. Meyer I. Prejudice, social stress, and mental health in lesbian, gay, and bisexual populations: Conceotual issues and research evidence. Psychol Bull 2003; 129 (5): 674-97.

5. Meyer I. Minority stress and mental health in gay men author. J Health Soc Behav 1995; 36 (1): 38-56.

6. Herek GM, Gillis JR, Cogan JC, Glunt EK. Hate Crime Victimization Among Lesbian, Gay, and Bisexual Adults: Prevalence, Psychological Correlates, and Methodological Issues. J Interpers Violence 1997; 12 (2): 195-215.

7. Huebner DM, Rebchook GM, Kegeles SM. Experiences of harassment, discrimination, and physical violence among young gay and bisexual men. Am J Public Health 2004; 94 (7): 1200-3.

8. Warner J, McKeown T, Griffin M, Johnson K, Ramsay a, Cort C, et al. Rates and predictors of mental illness in gay men, lesbians and bisexual men and women - Results from a survey based in England and Wales. 2004; Available from: http://discovery.ucl.ac.uk/6556/

9. Hamilton CJ, Mahalik JR. Minority stress, masculinity, and social norms predicting gay men's health risk behaviors. J Couns Psychol 2009; 56 (1): 132-41.

10. Hatzenbuehler ML, Mclaughlin KA, Nolen-hoeksema $\mathrm{S}$. Emotion regulation and internalizing symptoms in a longitudinal study of sexual minority and heterosexual adolescents. J Child Psychol Psychiatry Allied Discip 2008; 49 (12): 1270-8.

11. Pascoe EA, Richman LS. Perceived discrimination and health: A meta-analytil review. Psychol Bull 2009; 135 (4): 531-54.

12. Jarama SL, Kennamer JD, Poppen PJ, Hendricks M, Bradford J. Psychosocial, behavioral, and cultural predictors of sexual risk for HIV infection among latino men who have sex with men. AIDS Behav 2005; 9 (4): 513-23.

13. Fergusson DM, Horwood LJ, Beautrais AL. Is sexual orientation related to mental health problems and suicidality in young people? Arch Gen Psychiatry 1999; 56 (10): 876-80.

14. D'Augelli AR, Hershberger SL, Pilkington NW. Lesbian, gay, and bisexual youth and their families: Disclouse of sexual orientation and its consequences. Am J Orthopsychiatry 1998; 68 (3): 361-71.

15. Herek G, Gillis JR, Cogan J. Psychological Sequelae of Hate Crime Victimization Among Lesbian, Gay, and Bisexual Adults. J Consult Clin Psychol 1999; 67 (6): 945-51.

16. Burgess D, Tran A, Lee R, van Ryn M. Effects of perceived discrimination on mental health and mental health services utilization among gay, lesbian, bisexual and transgender persons. J LGBT Health Res 2007; 3 (4): $1-14$.

17. Sandfort TG, de Graaf R, Bijl RV, Schnabel P. Same-sex sexual behavior and psychiatric disorders: Findings from the Netherlands Mental Health Survey and Incidence Study (NEMESIS). Arch Gen Psychiatry 2001; 58 (1): 85-91.

18. King M, Semlyen J, Tai SS, Killaspy H, Osborn D, Popelyuk $\mathrm{D}$, et al. A systematic review of mental disorder, suicide, and deliberate self harm in lesbian, gay and bisexual people. BMC Psychiatry 2008; 8 (70): 1-17.

19. Cochran SD, Mays VM, Sullivan JG. Prevalence of mental disorders, psychological distress, and mental health services use among lesbian, gay, and bisexual adults in the United States. J Consult Clin Psychol 2003; 71 (1): 53-61.

20. Paz-Bailey G, Miller W, Shiraishi RW, Jacobson JO, Abimbola TO, Chen SY. Reaching men who have sex with men: a comparison of respondent-driven sampling and time-location sampling in Guatemala City. AIDS Behav 2013; 17 (9): 3081-90.

21. Barrientos J, Cárdenas M. Construction and validation of a subjective scale of stigma and discrimination (SISD) for the gay men and transgender women population in Chile. Sex Res Soc Policy 2014; 11 (3): 187-98.

22. Barrientos J, Cárdenas M, Gómez F. Características sociodemográficas, bienestar subjetivo y homofobia en una muestra de hombres gay en tres ciudades chilenas Socio-demographic characteristics, subjective well-being , and homophobia experienced by a sample of gay men from three cities in. Cad Saude Publica 2014; 30 (6): 1259-69.

23. Diener E, Emmons R A, Larsen RJ, Griffin S. The Satisfaction With Life Scale. J Pers Assess. 1985. p. 71-5.

24. Cárdenas M, Barrientos J, Bilbao M, Páez D, Gómez F, 
Asún D. Estructura factorial de la Escala de Satisfacción con la Vida en una muestra de estudiantes universitarios chilenos. Rev Mex Psicol 2012; 29 (2): 157.

25. Lambert MJ, Burlingame GM, Umphress V, Hansen N, Vermeersch DA, Clouse GC, et al. The reliability and validity of the Outcome Questionnaire. Clin Psychol Psychother 1996; 3 (4): 249-58.

26. De la Parra G, von Bergen A, del Río M. Primeros hallazgos de la aplicación de un instrumento que mide resultados psicoterapéuticos en una muestra de pacientes y de población general. Rev Chil Neuro-psiquiat 2002; 40 (3): 201-9.

27. Barrientos J, Meza P, Gómez F, Catalán S, Longueira J, Silva J. Política, derechos, violencia y diversidad sexual: primera encuesta Marcha por el Orgullo y Diversidad Sexual. Santiago: Chile: CLAM/IMS/UERJ; 2008.

28. Faul F, Erdfelder E, Lang AG, Buchner A. G*Power 3: A flexible statistical power analysis program for the social, behavioral, and biomedical sciences. Behav Res Methods 2007; 39 (2): 175-91.

29. Vera-Villarroel P, Celis-Atenas K, Córdova-Rubio N. Evaluación de la Felicidad: Análisis Psicométrico de la Escala de Felicidad Subjetiva en Población Chilena. Terapia psicológica 2011; 29 (1): 127-33.

30. Cárdenas M, Barrientos J. Homofobia y calidad de vida de gay y lesbianas: Una mirada psicosocial. Psykhe. 2013; 22 (1): 3-14.

31. Bahamondes-Correa J. System justification's opposite effects on psychological well-being: Testing a moderated mediation model in a gay men and lesbian sample in Chile. J Homosex 2016; 63 (11): 1537-55.

32. Guzmán-González M, Trabucco C, Urzúa A, Garrido L, Leiva J. Validez y confiabilidad de la versión adaptada al español de la Escala de Dificultades de Regulación Emocional (DERS-E) en población chilena. Terapia Psicológica 2014; 32 (1): 19-29.

33. León MJ, Chaigneau, S, del Río, P, Rodríguez, J. Sintomatología clínica en adolescentes chilenas heterosexuales y homosexuales. Rev GPU 2016; 12 (4): 413-9.

34. Belous CK, Wampler RS. Development of the gay and lesbian relationship satisfaction Scale. J Marital Fam Ther 2016; 42 (3): 451-65.

35. LaSala MC. Gay Male Couples The Importance of Coming Out and Being Out to Parents. J Homosex 2000; 39 (2): 47-71.

36. Savin-Williams RC. Coming out to parents and self-esteem Among gay and lesbians youths. J Homosex 1990; 18 (1-2): 1-35.

37. Tomicic A, Gálvez C, Quiroz C, Mertínez C, Fontbona J, Rodríguez J, et al. Suicidio en poblaciones lesbiana, gay, bisexual y trans: revisión sistemática de una década de investigación (2004-2014). Rev Med Chile 2016; 144 (6): 723-33. Disponible en: www.scielo.cl [Consultado el 4 de octubre de 2016].

38. Cárdenas M, Yáñez S. Nuevas formas de muestreo para minorías y poblaciones ocultas: muestras por encuestado conducido en una población de inmigrantes sudamericanos. Univ Psychol 2012; 11 (2): 571-8.

39. Muhib FB, Lin LS, Stueve A, Miller RL, Ford WL, Johnson WD, et al. A venue-based method for sampling hard-to-reach populations. Public Heal Rep 2001; 116 (1): 216-22.

40. Worthen MGF. An argument for separate analyses of attitudes toward lesbian, gay, bisexual men, bisexual women, MtF and FtM transgender individuals. Sex Roles 2013; 68 (11): 703-23. 